\title{
A Unified DC-AC Power Flow Algorithm of Distribution Network with Distributed Generation
}

\author{
Wenbo Li ${ }^{1, a, *}$, Keqiang Li ${ }^{2, b}$, Donglei Sun ${ }^{3}$, Lulu $\mathrm{Li}^{2}$, Chunlong Chen ${ }^{2}$, Si Yang \\ ${ }^{1}$ State Grid Shandong Electric Power Research Institute, Jinan, China \\ ${ }^{2}$ Key Laboratory of Power System Intelligent Dispatch and Control of Ministry of Education (Shandong \\ University), Jinan, China \\ ${ }^{3}$ Economic\&Technology Research Institute, State Grid Shandong Electric Power Company, Jinan, China \\ a liwenbo_1984@foxmail.com, ${ }^{\mathrm{b}}$ 18366110820@163.com
}

Keywords: DC-AC distribution network, voltage source converter, distributed generation, power flow, forward backward sweep method

\begin{abstract}
With the development of rectification technology and DC equipment, hybrid DC-AC transmission has arisen in the distribution network. In addition, the penetration of distributed generation (DG) in the distribution network makes the analysis of power system more complicated. As a key measure of grid programming and operating, a practical power flow method of DC-AC distribution network is urgent to study. Firstly, the mathematical model of DGs is given in this paper, and the analysis of the operation and control mode of DC-AC distribution system with voltage source converter (VSC) is conducted. Then a calculation method of hybrid DC-AC distribution system with DGs is proposed, in which constant-power DG, voltage controlled DG and DG with voltage static characteristic is concerned. An improved forward backward sweep method is given, concerning VSC, AC circuits, DC circuits and various type of DGs, which achieves the purpose of unified DC-AC power flow calculation. Finally, case study for a 90-node distribution network with DG integration is conducted to demonstrate the effectiveness of the method.
\end{abstract}

\section{Introduction}

With the increasing capacity of distributed energy resources and the substantial development of power electronic technology, a new distribution network featured with DGs, DC circuits, and DC loads draws more and more attention. Compared with traditional AC power grid, DC distribution network possesses better performance on such aspects as the acceptance of DGs, the transmission capacity, and the reliability of power supplies, and meanwhile, DC power grid can improve power quality effectively, reduce the use of power electronic equipment, and cut down the transmission loss of circuits ${ }^{[1]}$.

DGs including photovoltaic, wind power, internal combustion engine, micro gas turbine, fuel cell and so on, are characterized by low investment, high flexibility, perfect safety, and great reliability. Since different distributed power sources have different characteristics, it will be inconsistent with reality if such power sources are treated as active and reactive constant power sources during power flow calculation, which also affect the accuracy of the calculation results. Therefore, it is necessary to build mathematical models of different distributed power supplies and study corresponding flow calculation methods ${ }^{[3-4]}$.

With the development of power electronic technology and the mature application of full control devices, VSC demonstrates more and more significant advantages ${ }^{[2]}$. The problems faced by urban distribution systems will effectively solved by applying the DC technology based on the VSC to the area of supplying power via the distribution network. It has become an important research subject to conduct unified DC-AC power flow calculation on DC power distribution network containing VSC.

This paper introduces the modeling processes of unified DC-AC distribution network, VSC, and DGs. An improved forward backward sweep power flow algorithm is proposed, enabling the unified DC-AC power flow calculation containing VSC and DGs. The feasibility of the algorithm is 
proved through the calculation of a 90-node distribution system.

The remainder of this paper is organized as follows. Section 2 introduces the unified DC-AC distribution network with DGs. Section 3 introduces the mathematical model of DC-AC system. Section 4 shows the unified DC-AC power flow calculation method with DGs. And in the last section, case study is given to illustrate the feasibility of this method.

\section{Description of DC-AC System}

An DC-AC power system with DGs is shown in Fig.1. High voltage AC distribution network is connected to low voltage AC distribution network via transformer, or to DC distribution network via VSC. DGs are connected to the high voltage AC distribution network, low voltage AC distribution network or DC distribution network in a scattered manner.

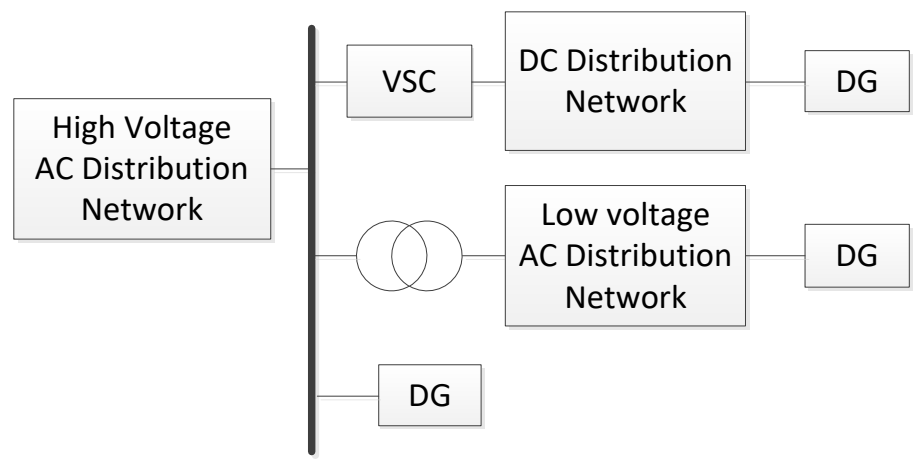

Fig.1 Unified DC-AC Distribution Network

DGs of different types is suitable for different types of grids. The power generated by such DG devices as photovoltaic and fuel cells is DC, which can be directly connected into DC power grid without conversion. The power generated by internal combustion engine and so on is AC, which can be integrated into AC power grid.

\section{Mathematical Model of DC-AC System}

\subsection{AC System}

Node $\mathrm{i}$ and node $\mathrm{j}$ represent two adjacent nodes in a radial distribution system. $U_{j}$ is the voltage of node $\mathrm{j}, S_{j}$ is the apparent power of node $\mathrm{j}$, then the current $I_{j}$ is deduced as

$$
\dot{I}_{j}=\frac{\tilde{S}_{j}^{*}}{\dot{U}_{j}^{*}}
$$

The power lost on the impedance $\mathrm{Z}$ between circuit $\mathrm{i}$ and circuit $\mathrm{j}$ is

$$
\Delta \tilde{S}=\left|\frac{S_{j}}{U_{j}}\right|^{2} Z
$$

Then the apparent power on node $\mathrm{i}$ is

$$
\tilde{S}_{i}=\tilde{S}_{j}+\left|\frac{S_{j}}{U_{j}}\right|^{2} Z
$$

(3) is the forward sweep formula of AC grid power.

$\Delta \dot{U}$ is the voltage loss on the impedance of the circuit, then the following relation can be obtained according to Ohm's Law

$$
\Delta \dot{U}=\dot{I} Z
$$

According to (1) and (4), the voltage of node $\mathrm{j}$ is as follows 


$$
\dot{U}_{j}=\dot{U}_{i}-\frac{\dot{S}_{i}^{*}}{\dot{U}_{i}^{*}} Z
$$

(5) is the backward sweep formula of AC grid voltage.

\subsection{System}

The forward backward sweep formula of DC system is similar to that of the AC system. The difference is that a DC system only has resistance without reactance and reactive power. The corresponding calculation formula is as follows.

The forward sweep formula of the power is as follows

$$
P_{i}=P_{j}+\left(\frac{P_{j}}{U_{j}}\right)^{2} R
$$

$P_{i}$ and $P_{j}$ are the power of node $\mathrm{i}$ and node $\mathrm{j}$ respectively, $U_{j}$ is the voltage of node $\mathrm{j}$, and $\mathrm{R}$ is the resistance of the circuit.

The backward sweep process of the voltage is as follows

$$
U_{j}=U_{i}-\frac{P_{i}}{U_{i}} R
$$

\subsection{VSC Model}

When the loss is ignored, the expression of the active power absorbed by VSC from the AC grid and that of the reactive power are as follows ${ }^{[5]}$ :

$$
\begin{gathered}
P_{s}=\frac{U_{s} U_{c}}{X} \sin \delta \\
Q_{s}=\frac{U_{s}\left(U_{s}-U_{c} \cos \delta\right)}{X}
\end{gathered}
$$

In the expressions, $U_{s}$ is the voltage amplitude of the AC grid, $U_{c}$ is the output voltage of the converter, $U_{d}$ is the voltage of the DC side, $\mathrm{X}$ is the equivalent reactance of VSC, and $\delta$ is the modulation angle.

The relation between $U_{c}$ and $U_{d}$ is as follows:

$$
U_{c}=\frac{M}{\sqrt{2}} U_{d}
$$

$\mathrm{M}$ is the modulation degree of the converter.

Since the loss of the converter is ignored, the active power on the AC and DC sides are equal and the expression is as follows:

$$
P_{s}=U_{d} I_{d}
$$

Since the power supply in the DC distribution network is weak in activity, the control modes of VSC are limited to the following two ${ }^{[6]}$ :

1) control by constant DC voltage $U_{d}$ and constant AC reactive power $Q_{V S C}$.

2) control by constant DC voltage $U_{d}$ and constant AC bus voltage $U$;

The loss of VSC can be expressed by the current and resistance of the converter, which is as follows

$$
P_{\text {loss }}=I^{2} R=\frac{P_{s}^{2}+Q_{s}{ }^{2}}{U_{s}^{2}} R
$$




\subsection{Distributed Generation Model}

\subsubsection{PQ Constant AC Distributed Generation}

DGs run by constant power factor can be expressed as this model. DGs of such devices as photovoltaic and micro gas turbines does not have automatic voltage regulation capacity and they run with constant power factor. PQ constant DGs can be regarded as load with negative sign, which is as (13).

$$
\left\{\begin{array}{l}
P=P_{s} \\
Q=Q_{s}
\end{array}\right.
$$

$P_{s}$ and $Q_{s}$ are the active and reactive values of constant power.

\subsubsection{AC Distributed Generation with Voltage Static Features}

DGs with voltage static characteristic usually have a constant active power output, while in the case of reactive power, it can be expressed as the function of the voltage which is as shown in (14). The reactive power sent or absorbed by such DGs is related to the voltage of the grid connection point. The asynchronous generator of wind power generation can be equivalent to such model. Meanwhile, as for DGs under reactive power-voltage droop control, the reactive power and the voltage are of a linear function relationship, which can also be equivalent to such model.

$$
\left\{\begin{array}{l}
P=P_{s} \\
Q=f(V)
\end{array}\right.
$$

$P_{s}$ is the constant active power of the power supply, and $f(V)$ is the voltage function of the reactive power of the DGs.

\subsubsection{PV Constant AC Distributed Generation}

The asynchronous generator with excitation regulation function can be expressed as this model ${ }^{[7-8]}$. As for photovoltaic converter with large reactive power capacity, a feedback control can be set to control the reactive power, and furthermore, the voltage of the grid connection point can be maintained at a constant value. Such DGs with voltage regulation function can be equivalent to a PV constant model. Its active power and voltage are constant, which is shown in (15).

$$
\left\{\begin{array}{l}
P=P_{s} \\
V=V_{s}
\end{array}\right.
$$

$P_{s}$ and $V_{s}$ are the constant active power and voltage value of the power supply.

\subsubsection{Distributed Generation}

The active power of the DC DGs is a constant value, which can be shown as (16)

$$
P=P_{d}
$$

$P_{d}$ is a constant value of the active power.

\section{DC-AC System Power Flow Algorithm}

\subsection{System}

Since VSC has the ability to control the voltage of the DC side, the DC distribution network and the AC distribution network can be calculated separately. As for the DC distribution network, since the DC side voltage of the VSC is constant, the iterative computation can be done according to (6) and (7) until the difference between the voltage of two adjacent iterations $U_{i}^{k+1}, U_{i}^{k}$ is smaller than the set value $\delta$, as shown in (17).

$$
\left|U_{i}^{k+1}-U_{i}^{k}\right|<\delta
$$




\subsection{VSC and AC System}

After the calculation convergence of the DC distribution network, the VSC and the DC distribution network are equivalent to a node of the AC system according to VSC control mode.

When the constant DC voltage $U_{d}$ constant AC reactive power $Q_{V S C}$ control mode is used, the active power on the AC side of the VSC is equal to the sum of the VSC loss and the VSC active power calculated through DC distribution network power flow algorithm. As the value of reactive power is set, the VSC is equivalent to a PQ node in the AC system.

When the constant DC voltage $U_{d}$ constant AC bus voltage $U_{s}$ control mode is used, the AC side active power of the VSC is calculated according to the DC distribution network power flow. Since the voltage is a set, the VSC is equivalent to a PV node of the AC system. The calculation method can refer to PV constant DGs.

After the equivalence of VSC and DC generation network, the AC system will be conducted with iterative calculation according to (3) and (5) until the difference between voltage results of two adjacent iterations is smaller than the set value.

\subsection{Distributed Generations}

\subsubsection{PQ Constant AC Distributed Generation}

PQ constant AC DGs and DC DGs share the completely same calculation method with traditional loads, but the symbols are opposite.

\subsubsection{Distributed Generation with Voltage Static Features}

As for the AC DGs with voltage static features, in every iteration the voltage value of the last iteration is used for the reactive power in the next iteration according to the functional relation.

\subsubsection{PV Constant AC Distributed Generation}

It is assumed that there are n PV nodes in the radial distribution system. The relationship between the reactive power of PV node $i$ and the voltage of PV node $j$ is linear, because when the voltage amplitude is constant, the injected reactive power of the node has a direct ratio with the injected current of the node. Since the current and the voltage meet the superposition theorem, the voltage undergoes linear change with reactive power eventually. The relation can be expressed as follows

$$
\Delta U_{j}=Z_{i j} \Delta Q_{i}
$$

The influence of the reactive power of the node on its own voltage is also linear:

$$
\Delta U_{i}=Z_{i i} \Delta Q_{i}
$$

Since there are $\mathrm{n}$ PV nodes interacting with each other, the relationship matrix is as follows.

$$
\left[\begin{array}{c}
\Delta U_{1} \\
\Delta U_{2} \\
\vdots \\
\Delta U_{n}
\end{array}\right]=\left[\begin{array}{cccc}
Z_{11} & Z_{12} & \cdots & Z_{1 n} \\
Z_{21} & Z_{22} & \cdots & Z_{2 n} \\
\vdots & \vdots & \ddots & \\
Z_{n 1} & Z_{n 2} & & Z_{n n}
\end{array}\right]\left[\begin{array}{c}
\Delta Q_{1} \\
\Delta Q_{2} \\
\vdots \\
\Delta Q_{n}
\end{array}\right]
$$

And it can be abbreviated as follows

$$
\Delta U=Z \Delta Q
$$

As for the formation of matrix $Z$, the values of $Z_{i j}$ can be obtained by deducing the relational expression of the voltage of a random node $i$ and a random node $j$ based on the theoretical knowledge of the circuit.

According to the difference between the set voltage of PV node and the voltage value in the iteration process, the corrected value for the next iteration on the reactive power of PV node is

$$
\Delta Q=Z^{-1} \Delta U
$$

A new value is given to the reactive power of PV node in the next iteration 


$$
Q^{k+1}=Q^{k}+\Delta Q
$$

\subsection{Power Flow Calculation Method}

The complete power flow calculation method is described as follows. The DC distribution network power flow is calculated first, after which, VSC and DC distribution networks are processed to be equivalent to nodes in the AC distribution network. Then, the AC distribution network is calculated, and every iteration step requires nesting the calculation of the distributed power. At last the power flow calculation process ends after the convergence of AC distribution network.

Flow chart of power flow calculation is shown in Fig.2.

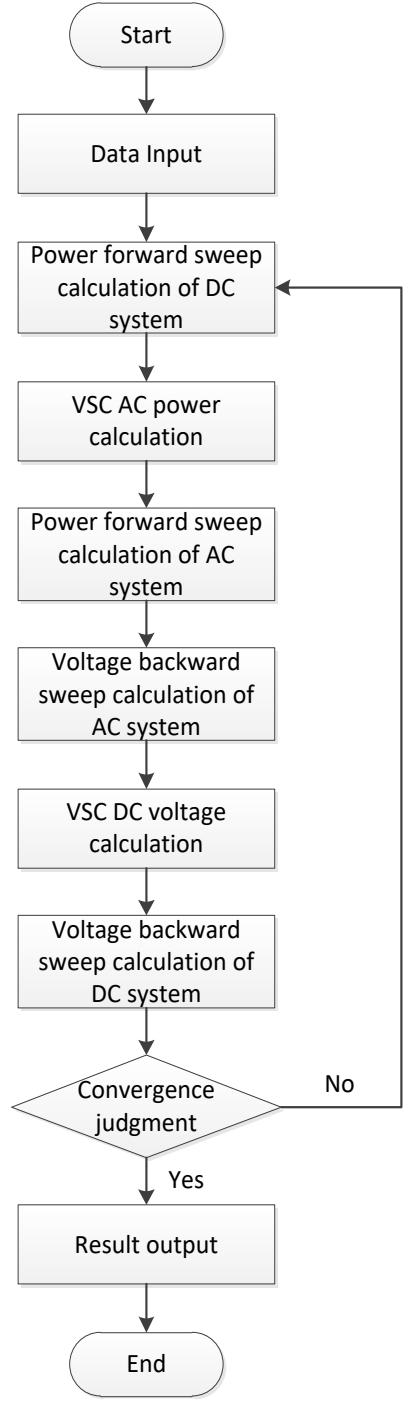

Fig.2 Flow Chart of Unified DC-AC Forward Backward Sweep Method

\section{Analysis on case study}

The case in this paper adopts the system combining AC and DC distribution networks. The converter adopts the VSC model shown in Section 3.3. DGs of four types shown in Section 3.4 are also included. The schematic diagram is shown in Fig.3. 




Fig.3 Radial Network Schematic Diagram

The error is set as $10^{\wedge}-4$, then the program achieves the convergence. The number of iterations is 4, which shows a good property of convergence. Convergence rate are shown as Fig.4.

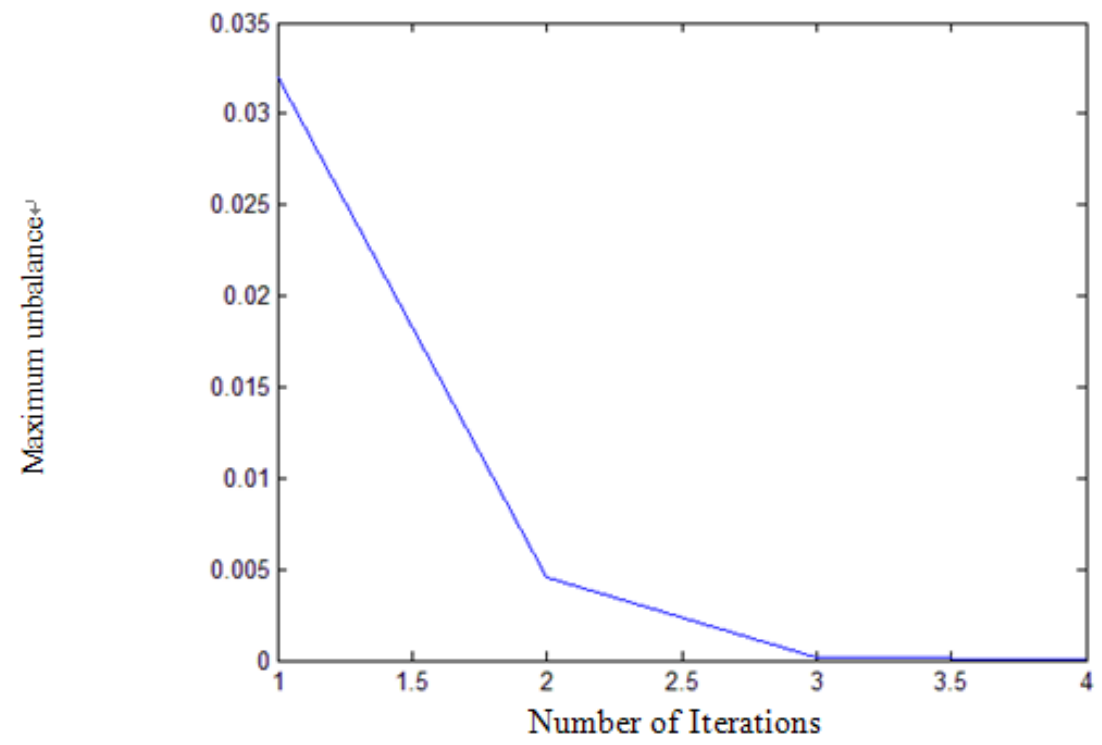

Fig.4 Convergence Rate of Forward Backward Sweep Method

In this paper, the influence of the addition of DC grid and DGs on the convergence of the program and the calculation speed is tested, with the results being listed in Table 1. 
Table 1 Influence of Addition of DC Elements on Program Performance

\begin{tabular}{|c|c|c|}
\hline Test Type & Number of Iterations & Calculation Time/s \\
\hline A & 3 & 0.0623 \\
\hline B & 3 & 0.0856 \\
\hline C & 3 & 0.0889 \\
\hline D & 4 & 0.0992 \\
\hline E & 4 & 0.0899 \\
\hline
\end{tabular}

Test types are,

A: 67-node AC system.

B: 67-node AC system and 23-node DC system

C: 67-node AC system, 23-node DC system and PQ-type DGs. Four PQ DGs are arranged at nodes $7,43,53$, and 62 .

D: 67-node AC system, 23-node DC system and PV-type DGs. Three PV DGs are arranged at nodes 31, 32, and 39.

E: 67-node AC system, 23-node DC system, $\mathrm{Q}=\mathrm{f}(\mathrm{V})$-type DGs. Three $\mathrm{Q}=\mathrm{f}(\mathrm{V}) \mathrm{DG}$ are arranged at nodes 14,35 , and 50.

According to the test results, since there is no big difference between the calculation methods, the addition of DC system has no big influence on the AC system, and both the convergence and the calculation rate are consistent with the calculation of pure AC system. PQ-type DGs has the same features with load, and its addition has the smallest influence on the operation of the program. PV-type DGs and $\mathrm{Q}=\mathrm{f}(\mathrm{V})$-type DGs have some influence on the program but not serious. Overall, this method shows good performance on convergence and calculation rate.

\section{Conclusion}

A unified DC-AC power flow algorithm of distribution network with DGs is shown in this paper. The calculation of AC grid and DC grid shows good performance on both calculation efficiency and convergence; the power flow algorithm containing several kinds of DGs is realized by making the DGs equivalent to different mathematical models. This method provides certain reference for the planning and analysis of the future distribution network.

\section{Reference}

[1]Jiang D.Z.,Zheng H.:'Research status and developing prospect of DC distribution network',Automation of Electric Power Systems， 2012， 36(8): 98-104.

[2]Flourentzou N., Agelidis V.G., Demetriades G.D. :'VSC based HVDC power transmission systems: an overview',IEEE Transactions on Power Electronics, 2009, 24(3): 592-602.

[3]Mohammadhafez B.,Nikolaos G.: 'Decentralized Stochastic Optimal Power Flow in Radial Networks With Distributed Generation’,IEEE Transactions on Smart GRID,2017,8(2):787-801.

[4]Jordan R.,Miroljub J.,Dardan K.: 'Optimal Power Flow for Distribution Networks with Distributed Generation’,Serbian Journal of Electrical Engineering,2017,12(2),145-170.

[5]Zhang X.P.: 'Multiterminal voltage-sourced converter based HVDC models for power flow analysis’,IEEE Transactions on Power Systems, 2004, 19(4): 1877-1884.

[6]Angeles-Camacho C., Tortelli O.L., Acha E., et al. 'Inclusion of a high voltage DC-voltage source converter model in a Newton-Raphson power flow algorithm',IEEE Proceedings-Generation Transmission and Distribution, 2003, 150(6): 691-696. 
[7]Pedram J.,Dionysios C.: ‘ A.Distributed Volt/VAr Control by PV Inverters’ ,IEEE Transaction on Power Systems,2013,28(3),3429-3439.

[8]Ángel M.,Rosa A. M.,Tania G.S.: 'Smart Inverter Volt/Var Control Functions forHigh Penetration of PV on Distribution Systems',IEEE Transactions on Smart Grid,2017,8(1),447-456. 